PROCEEDINGS OF THE

AMERICAN MATHEMATICAL SOCIETY

Volume 140, Number 11, November 2012, Pages 3715-3723

S 0002-9939(2012)11215-8

Article electronically published on March 7, 2012

\title{
NICHOLS ALGEBRAS ASSOCIATED TO THE TRANSPOSITIONS OF THE SYMMETRIC GROUP ARE TWIST-EQUIVALENT
}

\author{
L. VENDRAMIN \\ (Communicated by Gail R. Letzter)
}

\begin{abstract}
Using the theory of covering groups of Schur we prove that the two Nichols algebras associated to the conjugacy class of transpositions in $\mathbb{S}_{n}$ are equivalent by twist and hence they have the same Hilbert series. These algebras appear in the classification of pointed Hopf algebras and in the study of the quantum cohomology ring of flag manifolds.
\end{abstract}

\section{INTRODUCTION}

Nichols algebras play a fundamental role in the classification of finite-dimensional pointed Hopf algebras over $\mathbb{C}$. They are graded Hopf algebras

$$
\mathfrak{B}(V)=\bigoplus_{n \geq 0} \mathfrak{B}^{n}(V)=\mathbb{C} \oplus V \oplus \mathfrak{B}^{2}(V) \oplus \cdots
$$

in the category of Yetter-Drinfeld modules over a Hopf algebra $H$, and they are uniquely determined by $V$, the homogeneous component of $\mathfrak{B}(V)$ of degree one.

Let $H$ be the group algebra of a finite group $G$. In the study of Nichols algebras a basic question is to describe those Yetter-Drinfeld modules $V$ over $H$ for which $\mathfrak{B}(V)$ is finite-dimensional. Whereas deep results were found for the case where $G$ is abelian, [5, 16, 17, the situation is widely unknown for non-abelian groups $G$.

The first examples of finite-dimensional pointed Hopf algebras with non-abelian coradical appeared in [21, as bosonizations of Nichols algebras related to the transpositions in $\mathbb{S}_{3}$ and $\mathbb{S}_{4}$. The analogous Nichols algebra over $\mathbb{S}_{5}$ was computed by Graña; see 14. These Nichols algebras are computed from the conjugacy class of transpositions and a 2-cocycle (cocycle for short) associated to this conjugacy class. The cocycles arise from a cohomology theory defined for racks (see for example [3, 2, 13]). In [2], Theorem 1.1, it is proved that for all $n \in \mathbb{N}, n \geq 4$, there are precisely two rack 2-cocycles associated to the conjugacy class of transpositions in $\mathbb{S}_{n}$ that might have finite-dimensional Nichols algebras. Explicitly, one of these cocycles is the constant cocycle -1 . The other one is the cocycle given by

$$
\chi(\sigma, \tau)= \begin{cases}1 & \text { if } \sigma(i)<\sigma(j), \\ -1 & \text { if } \sigma(i)>\sigma(j)\end{cases}
$$

Received by the editors November 17, 2010 and, in revised form, February 9, 2011 and April 26, 2011.

2010 Mathematics Subject Classification. Primary 16T05, 16T30, 17 B37.

The author's work was partially supported by CONICET.

(C)2012 American Mathematical Society Reverts to public domain 28 years from publication 3715 
for all transpositions $\sigma$ and $\tau=(i j)$ with $i<j$. For all $n \in\{4,5\}$ the Nichols algebra associated to the conjugacy class of transpositions in $\mathbb{S}_{n}$ and any of the two cocycles $-1, \chi$ is finite-dimensional. Moreover, both of these algebras have the same Hilbert series. It is not known whether these algebras are finite-dimensional for $n>5$. The main result of this work is to connect these two algebras by twisting the cocycle. More precisely, we prove that the constant cocycle -1 and $\chi$ are equivalent by a twist. This gives an affirmative answer to a question due to Andruskiewitsch; see [1, Question 7. However, the problem arose already earlier in the literature. For example, in the last paragraph of [19], Majid discusses the relationship between these two algebras and the related quadratic algebras. To reach our main result, we use the existence of projective representations of $\mathbb{S}_{n}$. Projective representations of $\mathbb{S}_{n}$ were originally studied by Schur in 1911; see 22] for an English translation of his fundamental paper about this subject. As a corollary of our result we obtain that for all $n \geq 4$, both Nichols algebras associated to the conjugacy class of transpositions of $\mathbb{S}_{n}$ have the same Hilbert series.

We recall briefly another application for Nichols algebras which may have connections with the main result of this work. In [8], Borel identified the cohomology ring of a flag manifold with $S_{W}$, the algebra of coinvariants of the associated Coxeter group $W$. This admits certain divided-difference operators which create classes of Schubert manifolds. In [11, Fomin and Kirillov introduced a new model for the Schubert calculus of a flag manifold, realizing $S_{W}$ as a commutative subalgebra of a non-commutative quadratic algebra $\mathcal{E}_{W}$, when $W$ is a symmetric group. In [6], Bazlov proved that Nichols algebras provide the correct setting for this model for Schubert calculus on a flag manifold. It is an open problem whether the Nichols algebra associated to $\chi$ coincides with the quadratic algebra $\mathcal{E}_{W}[19,21]$.

\section{Preliminaries}

2.1. Racks and cohomology. We briefly recall basic facts about racks; see [3] for more information and references.

A rack is a pair $(X, \triangleright)$ where $X$ is a non-empty set and $\triangleright: X \times X \rightarrow X$ is a function, such that the map $x \mapsto i \triangleright x$ is bijective for all $i \in X$, and $i \triangleright(j \triangleright k)=$ $(i \triangleright j) \triangleright(i \triangleright k)$ for all $i, j, k \in X$. A subrack of $X$ is a non-empty subset $Y \subseteq X$ such that $(Y, \triangleright)$ is also a rack.

Example 2.1. A group $G$ is a rack with $x \triangleright y=x y x^{-1}$ for all $x, y \in G$. If a subset $X \subseteq G$ is stable under conjugation by $G$, then it is a subrack of $G$. In particular, the conjugacy class of transpositions in $\mathbb{S}_{n}$ is a rack; it will be denoted by $X_{n}$.

In this work we are interested only in racks which can be realized as a finite conjugacy class of a group. Let $X$ be such a rack. A map $q: X \times X \rightarrow \mathbb{C}^{\times}$is a 2 -cocycle if and only if

$$
q_{x, y \triangleright z} q_{y, z}=q_{x \triangleright y, x \triangleright z} q_{x, z}
$$

for all $x, y, z \in X$. We write $Z_{R}^{2}\left(X, \mathbb{C}^{\times}\right)$for the set of all rack 2-cocycles. Let $q, q^{\prime} \in Z_{R}^{2}\left(X, \mathbb{C}^{\times}\right)$. We write $q \sim q^{\prime}$ if there exists $\gamma: X \rightarrow \mathbb{C}^{\times}$such that

$$
q_{x, y}=\gamma_{x \triangleright y}^{-1} q_{x, y}^{\prime} \gamma_{y}
$$

for all $x, y \in X$. Since $\sim$ is an equivalence relation and $Z_{R}^{2}\left(X, \mathbb{C}^{\times}\right)$is stable under $\sim$ it is possible to define the second rack cohomology group as

$$
H_{R}^{2}\left(X, \mathbb{C}^{\times}\right)=Z_{R}^{2}\left(X, \mathbb{C}^{\times}\right) / \sim
$$


All these notions are based on the abelian cohomology theory of racks proposed independently in [9], 13]. For more details about cohomology theories of racks, see [3],$\$ 4$.

2.2. Nichols algebras. We refer to 4 for an introduction to Yetter-Drinfeld modules and Nichols algebras.

Let $n \in \mathbb{N}$. We recall the well-known presentation of the braid group $\mathbb{B}_{n}$ by generators and relations. The group $\mathbb{B}_{n}$ has generators $\sigma_{1}, \ldots, \sigma_{n-1}$ and relations

$$
\begin{aligned}
\sigma_{i} \sigma_{j} \sigma_{i}=\sigma_{j} \sigma_{i} \sigma_{j} & \text { for }|i-j|=1 \\
\sigma_{i} \sigma_{j}=\sigma_{j} \sigma_{i} & \text { for }|i-j|>1 .
\end{aligned}
$$

There exists a canonical projection $\mathbb{B}_{n} \rightarrow \mathbb{S}_{n}$ that admits the so-called Matsumoto section $\mu: \mathbb{S}_{n} \rightarrow \mathbb{B}_{n}$ such that $\mu((i i+1))=\sigma_{i}$. This section satisfies the following: $\mu(x y)=\mu(x) \mu(y)$ for any $x, y \in \mathbb{S}_{n}$ such that $l(x y)=l(x)+l(y)$, where $l$ is the length function on $\mathbb{S}_{n}$.

Let $V$ be a vector space over $\mathbb{C}$ and let $c \in \mathbf{G L}(V \otimes V)$. The map $c$ is a solution of the braid equation if and only if

$$
(c \otimes \mathrm{id})(\mathrm{id} \otimes c)(c \otimes \mathrm{id})=(\mathrm{id} \otimes c)(c \otimes \mathrm{id})(\mathrm{id} \otimes c) .
$$

If $c$ is a solution of the braid equation, we say that $(V, c)$ is a braided vector space. A solution of the braid equation induces a representation

$$
\rho_{n}: \mathbb{B}_{n} \rightarrow \mathbf{G L}\left(V^{\otimes n}\right)
$$

defined by

$$
\rho_{n}\left(\sigma_{i}\right)=\mathrm{id}^{\otimes(i-1)} \otimes c \otimes \mathrm{id}^{\otimes(n-i-1)}
$$

for $1 \leq i \leq n-1$. Let

$$
Q_{n}=\sum_{\sigma \in \mathbb{S}_{n}} \rho_{n}(\mu(\sigma)): V^{\otimes n} \rightarrow V^{\otimes n}
$$

for $n \geq 2, Q_{0}=\operatorname{id}_{\mathbb{C}}$ and $Q_{1}=\operatorname{id}_{V}$. The Nichols algebra associated to the braided vector space $V$ is

$$
\mathfrak{B}(V)=T(V) / \bigoplus_{n \geq 2} \operatorname{ker} Q_{n} \simeq \bigoplus_{n \geq 0} \operatorname{im} Q_{n} .
$$

By [3, Theorem 4.14, Yetter-Drinfeld modules over group algebras can also be studied in terms of racks and rack 2-cocycles. Therefore we are interested in Nichols algebras of braided vector spaces arising from racks and 2-cocycles.

Let $(X, \triangleright)$ be a rack and let $q \in Z_{R}^{2}\left(X, \mathbb{C}^{\times}\right)$. We consider $V=\mathbb{C} X$, the vector space with basis $x \in X$, and define $c: V \otimes V \rightarrow V \otimes V$ as

$$
c(x \otimes y)=q_{x, y} x \triangleright y \otimes x .
$$

Then $c$ is a solution of the braid equation. The Nichols algebra associated to the pair $(X, q)$ is the Nichols algebra of the braided vector space $(V, c)$. This algebra will be denoted by $\mathfrak{B}(X, q)$.

Recall that $X_{n}$ is defined as the rack associated to the conjugacy class of transpositions in $\mathbb{S}_{n}$. In [2], Theorem 1.1, it is proved that there are two rack 2-cocycles associated to $X_{n}$ that might have a finite-dimensional Nichols algebra. One is the constant 2-cocycle -1 . The other is the 2-cocycle $\chi$ given by Equation (1). 
Remark 2.2. It can be checked directly that the 2-cocycles -1 and $\chi$ associated to the rack $X_{3}$ are cohomologous. Then the Nichols algebras $\mathfrak{B}\left(X_{3}, \chi\right)$ and $\mathfrak{B}\left(X_{3},-1\right)$ are isomorphic and hence they have the same Hilbert series.

For all $m \in \mathbb{N}$ let $(m)_{t}=1+t+\cdots+t^{m-1} \in \mathbb{Z}[t]$.

Example 2.3. The Nichols algebras $\mathfrak{B}\left(X_{4},-1\right)$ and $\mathfrak{B}\left(X_{4}, \chi\right)$ both have dimension 576. In both cases the Hilbert series is

$$
\mathcal{H}_{4}(t)=(2)_{t}^{2}(3)_{t}^{2}(4)_{t}^{2} .
$$

These algebras appeared first in [11,21]. For more information about these algebras, see [12, Theorem 2.4 and Proposition 2.5, or [15, Proposition 5.11.

Example 2.4. The Nichols algebras $\mathfrak{B}\left(X_{5},-1\right)$ and $\mathfrak{B}\left(X_{5}, \chi\right)$ both have dimension 8294400. In both cases the Hilbert series is

$$
\mathcal{H}_{5}(t)=(4)_{t}^{4}(5)_{t}^{2}(6)_{t}^{4} .
$$

These algebras were first computed by Graña [14. For more information about these algebras, see [12, Theorem 2.4 and Proposition 2.5, or [15], Proposition 5.13.

2.3. Twisting. In 1, Section 3.4 it is shown how to relate two rack 2-cocycles by a twisting in such a way that some properties of the corresponding Nichols algebras are preserved. This method is based on the twisting method of [10] and its relationship with the bosonization given in [20.

Let $X$ be a subrack of a conjugacy class of a group $G$. Let $q$ be a rack 2-cocycle on $X$ and let $\phi$ be a group 2-cocycle on $G$. Define $q^{\phi}: X \times X \rightarrow \mathbb{C}^{\times}$by

$$
q_{x, y}^{\phi}=\phi(x, y) \phi(x \triangleright y, x)^{-1} q_{x, y}
$$

for $x, y \in X$.

Remark 2.5. Let $X$ be a rack and $q \in H_{R}^{2}\left(X, \mathbb{C}^{\times}\right)$. For a map $\phi: X \times X \rightarrow \mathbb{C}^{\times}$, define $q^{\phi}$ by Equation (21). Then $q^{\phi}$ is a rack 2-cocycle if and only if

$$
\begin{aligned}
\phi(x, z) \phi(x \triangleright y, x \triangleright z) \phi(x \triangleright(y \triangleright z), x) \phi(y \triangleright z, y) & \\
& =\phi(y, z) \phi(x, y \triangleright z) \phi(x \triangleright(y \triangleright z), x \triangleright y) \phi(x \triangleright z, x)
\end{aligned}
$$

for any $x, y, z \in X$. Thus, if $X$ is a subrack of a group $G$ and $\phi$ is a group 2-cocycle, $\phi \in Z^{2}\left(G, \mathbb{C}^{\times}\right)$, then $\left.\phi\right|_{X \times X}$ satisfies equation (3). See [1], Remark 3.10, or 9], Theorem 7.1, for a similar result.

Lemma 2.6. The Hilbert series of $\mathfrak{B}(X, q)$ and $\mathfrak{B}\left(X, q^{\phi}\right)$ are equal.

Proof. See [1], Section 3.4.

Definition 2.7. The 2-cocycles $q$ and $q^{\prime}$ on $X$ are equivalent by twist if there exists $\phi: X \times X \rightarrow \mathbb{C}^{\times}$such that $q^{\prime}=q^{\phi}$ as in (2).

\section{The Schur COVER OF $\mathbb{S}_{n}$}

3.1. Projective representations and covering groups. We review some aspects of Schur's theory of projective representations and construct the Schur cover of $\mathbb{S}_{n}$. See [7, 18,22, for details.

A projective representation of a finite group $G$ is a group homomorphism $G \rightarrow$ $\operatorname{PGL}(V)$. Equivalently, such a representation may be viewed as a map $f: G \rightarrow$ $\mathbf{G L}(V)$ such that

$$
f(x) f(y)=\phi(x, y) f(x y)
$$


for all $x, y \in G$ and suitable scalars $\phi(x, y) \in \mathbb{C}^{\times}$. The map $G \times G \rightarrow \mathbb{C}^{\times}$, $(x, y) \mapsto \phi(x, y)$, is called a factor set. The associativity of the group $\mathbf{G L}(V)$ implies the 2-cocycle condition of the factor set $\phi$ :

$$
\phi(x, y) \phi(x y, z)=\phi(x, y z) \phi(y, z)
$$

for all $x, y, z \in G$.

Two projective representations $\rho_{1}: G \rightarrow \mathbf{G L}\left(V_{1}\right)$ and $\rho_{2}: G \rightarrow \mathbf{G L}\left(V_{2}\right)$ are equivalent if there exists a $\mathbb{C}$-linear isomorphism $S: V_{1} \rightarrow V_{2}$ and a map $b: G \rightarrow \mathbb{C}^{\times}$ such that

$$
b(x) S \rho_{1}(x) S^{-1}=\rho_{2}(x)
$$

for all $x \in G$. Two factor sets $\phi$ and $\phi^{\prime}$ are equivalent if they differ only by a factor $b_{x} b_{y} / b_{x y}$ for some $b: G \rightarrow \mathbb{C}^{\times}$. The Schur multiplier of $G$ is the abelian group of factor sets modulo equivalence. It is isomorphic to the second cohomology group $H^{2}\left(G, \mathbb{C}^{\times}\right)$.

Recall that a central extension of $G$ is a pair $(E, p)$, where $p: E \rightarrow G$ is a surjective group homomorphism such that $\operatorname{ker} p$ is contained in the center of the group $E$. Schur proved that every finite group $G$ has a central extension $(E, p)$ with the property that every projective representation $\rho$ of $G$ lifts to an ordinary representation $\bar{\rho}$ of $E$ such that the diagram

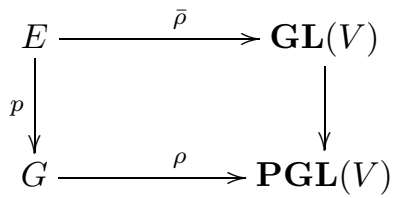

commutes.

There exist extensions with $\operatorname{ker} p \simeq H^{2}\left(G, \mathbb{C}^{\times}\right)$. Moreover, $H^{2}\left(G, \mathbb{C}^{\times}\right)$is the unique minimal possibility for $\operatorname{ker} p$. These minimal central extensions of $G$ are called Schur covering groups of $G$.

We recall that $\mathbb{S}_{n}$ has a Coxeter group presentation

$$
\begin{aligned}
\mathbb{S}_{n}=\left\langle s_{1}, \ldots, s_{n-1}\right| s_{i}^{2}=\left(s_{j} s_{j+1}\right)^{3}= & \left.\left(s_{k} s_{l}\right)^{2}=1\right\rangle \\
& (1 \leq i \leq n-1,1 \leq j \leq n-2, k \leq l-2),
\end{aligned}
$$

where $s_{1}, \ldots, s_{n-1}$ denote the adjacent transpositions of $\mathbb{S}_{n}$ defined by

$$
s_{1}=(12), s_{2}=(23), \ldots, s_{n-1}=(n-1 n) .
$$

Theorem 3.1. Given $n \geq 4$, define the group $T_{n}$ as follows:

$$
\begin{aligned}
T_{n}=\left\langle z, t_{1}, \ldots, t_{n-1} \mid t_{i}^{2}=\left(t_{j} t_{j+1}\right)^{3}=1,\left(t_{k} t_{l}\right)^{2}=z, z^{2}=\left[z, t_{i}\right]=1\right\rangle \\
(1 \leq i \leq n-1,1 \leq j \leq n-2, k \leq l-2) .
\end{aligned}
$$

Then $T_{n}$ is a Schur covering group of $\mathbb{S}_{n}$. Therefore, there exists a central extension

$$
0 \rightarrow A \stackrel{i}{\rightarrow} T_{n} \stackrel{p}{\rightarrow} \mathbb{S}_{n} \rightarrow 1
$$

where $A=\langle z\rangle$.

Proof. See [18], Theorem 2.12.3. 
3.2. Transpositions in $T_{n}$. We want to study the lifting of the conjugacy class of transpositions in $\mathbb{S}_{n}$. See [23], Section 3 for a detailed description about permutations in $T_{n}$.

Remark 3.2. Let $t \in T_{n}$. For any $\sigma \in \mathbb{S}_{n}$ we have that $p^{-1}(\sigma)=\{\bar{\sigma}, \bar{\sigma} z\}$. Since the involution $z$ is a central element of $T_{n}$, the group $\mathbb{S}_{n}$ acts on $T_{n}$ by conjugation:

$$
\sigma \triangleright t=\bar{\sigma} t(\bar{\sigma})^{-1}=(\bar{\sigma} z) t(\bar{\sigma} z)^{-1} .
$$

Therefore it is possible to write the conjugation in $T_{n}$ as $\sigma \triangleright t=\sigma t \sigma^{-1}$, where $t \in T_{n}$ and $\sigma \in \mathbb{S}_{n}$.

Definition 3.3. For $i, j \in \mathbb{N}$ such that $1 \leq i, j \leq n, i \neq j$, let $[i j]$ be an element of $T_{n}$ defined inductively as

$$
\begin{aligned}
{[i i+1] } & =t_{i}, \\
{[i j] } & =t_{i} \triangleright[i+1 j] z, \quad \text { for } i+1<j, \\
{[j i] } & =[i j] z .
\end{aligned}
$$

Lemma 3.4. Let $i, j, k \in\{1, \ldots, n\}$. Then $s_{k} \triangleright[i j]=\left[s_{k}(i) s_{k}(j)\right] z$.

Proof. Multiplying both sides by $z$ if needed, we may assume that $i<j$. If $\{k, k+1\} \cap\{i, j\}=\emptyset$, then the claim follows from [22, Paragraph 6, III. If $k=i-1$, then the claim follows from Definition 3.3 . The case $k=i$ follows from the case $k=i-1$ by applying $s_{i-1}$. Since $s_{j} \triangleright t_{j-1}=s_{j-1} \triangleright t_{j}$, a straightforward computation settles the case $k=j$. Finally, the case $k=j-1$ follows from the case $k=j$ by applying $s_{j}$.

Proposition 3.5. Let $l \in \mathbb{N}, \sigma=s_{i_{1}} s_{i_{2}} \cdots s_{i_{l}} \in \mathbb{S}_{n}$ and $i, j \in\{1, \ldots, n\}$. Then

$$
\sigma \triangleright[i j]=[\sigma(i) \sigma(j)] z^{l} .
$$

Proof. This follows from Lemma 3.4 by induction on $l$.

3.3. Nichols algebras over symmetric groups. Recall that $X_{n}$ is the rack of transpositions in $\mathbb{S}_{n}$. There exist two rack 2-cocycles that we want to consider. One of these rack 2-cocycles is the constant cocycle -1 . The other one is the 2-cocycle given by equation (11).

Lemma 3.6. Let $A$ be an abelian group and let $\psi: A \rightarrow \mathbb{C}^{\times}$be a group homomorphism. For $\phi \in Z^{2}\left(\mathbb{S}_{n}, A\right)$, define $\phi_{\psi}(x, y)=\psi(\phi(x, y))$. Then $\phi_{\psi} \in Z^{2}\left(\mathbb{S}_{n}, \mathbb{C}^{\times}\right)$.

Proof. This follows from [7], Chapter 6, §3.

Lemma 3.7. There exists a section $s: \mathbb{S}_{n} \rightarrow T_{n}$ such that if $\tau=(i j), i<j$, then

$$
s(\sigma) \triangleright s(\tau)= \begin{cases}s(\sigma \triangleright \tau) z & \text { if } \sigma(i)<\sigma(j), \\ s(\sigma \triangleright \tau) & \text { if } \sigma(i)>\sigma(j)\end{cases}
$$

for all $\sigma$. 
Proof. By Theorem 3.1 there exists a central extension

$$
0 \rightarrow A \stackrel{i}{\rightarrow} T_{n} \stackrel{p}{\rightarrow} \mathbb{S}_{n} \rightarrow 1
$$

where $A=\langle z\rangle$. Take any set-theoretical section $\bar{s}: \mathbb{S}_{n} \rightarrow T_{n}$ such that $\bar{s}(\mathrm{id})=1$ and define a map $s: \mathbb{S}_{n} \rightarrow T_{n}$ by

$$
s(\pi)= \begin{cases}\bar{s}(\pi) & \text { if } \pi \notin X_{n}, \\ {[i j]} & \text { if } \pi=(i j) \in X_{n}, \text { with } i<j .\end{cases}
$$

Then $p s=$ id and $s(\mathrm{id})=1$. Since $\sigma \in X_{n}$, the length of $\sigma$ is 1 . Remark 3.2 and Proposition 3.5 imply that

$$
s(\sigma) \triangleright s(\tau)=\sigma \triangleright s(\tau)=\sigma[i j] \sigma^{-1}=[\sigma(i) \sigma(j)] z .
$$

Hence the claim follows.

Theorem 3.8. Let $n \geq 4$. The rack 2-cocycles $\chi$ and -1 associated to $X_{n}$ are twist equivalent. Hence the Hilbert series of $\mathfrak{B}\left(X_{n},-1\right)$ and $\mathfrak{B}\left(X_{n}, \chi\right)$ are equal.

Proof. By Theorem 3.1 there exists a central extension

$$
0 \rightarrow A \stackrel{i}{\rightarrow} T_{n} \stackrel{p}{\rightarrow} \mathbb{S}_{n} \rightarrow 1
$$

where $A=\langle z\rangle$. Let $s: \mathbb{S}_{n} \rightarrow T_{n}$ be the section of Lemma 3.7 and let $\phi(x, y) \in A$ be defined by the equation

$$
s(x) s(y)=i(\phi(x, y)) s(x y) .
$$

Then $\phi \in Z^{2}\left(\mathbb{S}_{n}, A\right)$. For any group homomorphism $\psi: A \rightarrow \mathbb{C}^{\times}$apply Lemma 3.6 to get a group 2-cocycle $\phi_{\psi} \in Z^{2}\left(\mathbb{S}_{n}, \mathbb{C}^{\times}\right)$. Take $\psi=\operatorname{sgn}$, where $\psi(z)=-1$. We claim that

$$
-1=\phi_{\psi}(\sigma, \tau) \phi_{\psi}(\sigma \triangleright \tau, \sigma)^{-1} \chi(\sigma, \tau)
$$

for all $\sigma, \tau \in X_{n}$. In fact,

$$
\begin{aligned}
\phi_{\psi}(\sigma, \tau) & \phi_{\psi}(\sigma \triangleright \tau, \sigma)^{-1} \\
& \left.=\psi\left(s(\sigma) s(\tau) s(\sigma \tau)^{-1}\right) \psi\left(s\left(\sigma \tau \sigma^{-1}\right) s(\sigma) s(\sigma \tau)^{-1}\right)\right)^{-1} \\
& =\psi\left(s(\sigma) s(\tau) s(\sigma \tau)^{-1}\left(s\left(\sigma \tau \sigma^{-1}\right) s(\sigma) s(\sigma \tau)^{-1}\right)^{-1}\right) \\
& =\psi\left(s(\sigma) s(\tau) s(\sigma \tau)^{-1} s(\sigma \tau) s(\sigma)^{-1} s\left(\sigma \tau \sigma^{-1}\right)^{-1}\right) \\
& =\psi\left(s(\sigma) s(\tau) s(\sigma)^{-1} s\left(\sigma \tau \sigma^{-1}\right)^{-1}\right) .
\end{aligned}
$$

Therefore the first claim follows from Lemma 3.7. For the second claim, use Lemma 2.6 


\section{ACKNOWLEDGEMENTS}

The author is grateful to Matías Graña for valuable comments and discussions and to Nicolás Andruskiewitsch for useful discussions and for suggesting this project to him. The author thanks István Heckenberger for his careful reading of this work and also thanks the referee for suggesting Lemma 3.4 which simplified the original presentation of the paper.

\section{REFERENCES}

[1] N. Andruskiewitsch, F. Fantino, G. A. García, and L. Vendramin, On Nichols algebras associated to simple racks, Contemp. Math. vol. 537, Amer. Math. Soc., Providence, RI, 2011, $31-56$.

[2] N. Andruskiewitsch, F. Fantino, M. Graña, and L. Vendramin, Finite-dimensional pointed Hopf algebras with alternating groups are trivial, Ann. Mat. Pura Appl. (4) 190 (2011), no. 2, 225-245. MR2786171

[3] N. Andruskiewitsch and M. Graña, From racks to pointed Hopf algebras, Adv. Math. 178 (2003), no. 2, 177-243. MR 1994219(2004i:16046)

[4] N. Andruskiewitsch and H.-J. Schneider, Pointed Hopf algebras, New directions in Hopf algebras, Math. Sci. Res. Inst. Publ., vol. 43, Cambridge Univ. Press, Cambridge, 2002, pp. 1-68. MR.1913436 (2003e:16043)

[5] - On the classification of finite-dimensional pointed Hopf algebras, Ann. of Math. (2) 171 (2010), no. 1, 375-417. MR2630042 (2011j:16058)

[6] Y. Bazlov, Nichols-Woronowicz algebra model for Schubert calculus on Coxeter groups, J. Algebra 297 (2006), no. 2, 372-399. MR2209265 (2007b:20083)

[7] Ya. G. Berkovich and E. M. Zhmud', Characters of finite groups. Part 1, Translations of Mathematical Monographs, vol. 172, American Mathematical Society, Providence, RI, 1998. MR:1486039 (98m:20011)

[8] A. Borel, Sur la cohomologie des espaces fibrés principaux et des espaces homogènes de groupes de Lie compacts, Ann. of Math. (2) 57 (1953), 115-207 (French). MR0051508 $(14: 490 \mathrm{e})$

[9] J. S. Carter, D. Jelsovsky, S. Kamada, L. Langford, and M. Saito, Quandle cohomology and state-sum invariants of knotted curves and surfaces, Trans. Amer. Math. Soc. 355 (2003), no. 10, 3947-3989. MR1990571 (2005b:57048)

[10] Y. Doi and M. Takeuchi, Multiplication alteration by two-cocycles-the quantum version, Comm. Algebra 22 (1994), no. 14, 5715-5732. MR1298746 (95j:16043)

[11] S. Fomin and A. N. Kirillov, Quadratic algebras, Dunkl elements, and Schubert calculus, Advances in geometry, Progr. Math., vol. 172, Birkhäuser Boston, Boston, MA, 1999, pp. 147182. MR.1667680 (2001a:05152)

[12] G. A. García and A. García Iglesias, Finite dimensional pointed Hopf algebras over $\mathbb{S}_{4}$, Israel J. Math. 183 (2011), 417-444. MR2811166

[13] M. Graña, On Nichols algebras of low dimension, New trends in Hopf algebra theory (La Falda, 1999), Contemp. Math., vol. 267, Amer. Math. Soc., Providence, RI, 2000, pp. 111-134.

[14] _ Nichols algebras of non-abelian group type: zoo of examples. Web page available at http://mate.dm.uba.ar/ ${ }^{\text {matiasg/zoo. }}$

[15] M. Graña, I. Heckenberger, and L. Vendramin, Nichols algebras of group type with many quadratic relations, Adv. Math. 227 (2011), no. 5, 1956-1989. MR.2803792

[16] I. Heckenberger, The Weyl groupoid of a Nichols algebra of diagonal type, Invent. Math. 164 (2006), no. 1, 175-188. MR2207786 (2007e:16047)

[17] Classification of arithmetic root systems, Adv. Math. 220 (2009), no. 1, 59-124. MR2462836 (2009k:17017)

[18] G. Karpilovsky, The Schur multiplier, London Mathematical Society Monographs. New Series, vol. 2, The Clarendon Press, Oxford University Press, New York, 1987. MR1200015 (93j:20002)

[19] S. Majid, Noncommutative differentials and Yang-Mills on permutation groups $S_{n}$, Hopf algebras in noncommutative geometry and physics, Lecture Notes in Pure and Appl. Math., vol. 239, Dekker, New York, 2005, pp. 189-213. MR2106930 (2006b:58007) 
[20] S. Majid and R. Oeckl, Twisting of quantum differentials and the Planck scale Hopf algebra, Comm. Math. Phys. 205 (1999), no. 3, 617-655. MR.1711340 (2000h:58018)

[21] A. Milinski and H.-J. Schneider, Pointed indecomposable Hopf algebras over Coxeter groups, New trends in Hopf algebra theory (La Falda, 1999), Contemp. Math., vol. 267, Amer. Math. Soc., Providence, RI, 2000, pp. 215-236. MR.1800714 (2001k:16074)

[22] J. Schur, On the representation of the symmetric and alternating groups by fractional linear substitutions, Internat. J. Theoret. Phys. 40 (2001), no. 1, 413-458. Translated from the German [J. Reine Angew. Math. 139 (1911), 155-250] by Marc-Felix Otto. MR 1820589 (2003a:20016)

[23] R. A. Wilson, The finite simple groups, Graduate Texts in Mathematics, vol. 251, SpringerVerlag, London Ltd., London, 2009. MR2562037 (2011e:20018)

Departamento de Matemática - FCeyn, Universidad de Buenos Aires, Pab. I - Ciudad Universitaria (1428), Buenos Aires, Argentina

E-mail address: lvendramin@dm.uba.ar 